\title{
Diagnostic value and prognostic significance of CTRP9 combined with pentraxin-3 in acute coronary syndrome
}

\author{
NA JIANG $^{1 *}$, SHULONG ZHOU ${ }^{1 *}$, GUANGLEI WANG ${ }^{1}$, NINGNING JIANG ${ }^{2}$, \\ HUAIXIN WANG ${ }^{1}$ and FENG ZHAO ${ }^{3}$
}

\author{
Departments of ${ }^{1}$ Emergency and ${ }^{2}$ Ophthalmology, Yidu Central Hospital of Weifang, Weifang, Shandong 262500; \\ ${ }^{3}$ Department of Cardiology, Dongying Hospital of Traditional Chinese Medicine, Dongying, Shandong 257000, P.R. China
}

Received April 15, 2020; Accepted November 11, 2020

DOI: $10.3892 / \mathrm{etm} .2021 .9685$

\begin{abstract}
The present study aimed to explore the diagnostic value and prognostic significance of $\mathrm{Clq} /$ tumor necrosis factor-related protein 9 (CTRP9) combined with pentraxin-3 (PTX-3) in acute coronary syndrome (ACS). A total of 137 patients with coronary heart disease and chest pain were included. Among them, seventy-nine patients with ACS were allocated into a study group and fifty-eight patients with non-cardiac chest pain (NCCP) were allocated into a control group. The serum CTRP9, PTX-3 levels were quantified by ELISA, and their correlation with other ACS-related indexes, diagnostic value for ACS and predictive significance for poor prognosis were analyzed. In addition, the risk factors of the poor prognosis of ACS patients were studied. CTRP9 was lowly expressed and PTX-3 was highly expressed in the serum of ACS patients. CTRP9 was negatively correlated with cardiac troponin I (cTnI), creatine kinase-MB (CK-MB) and high-sensitivity $\mathrm{C}$-reactive protein (hs-CRP) $(\mathrm{P}<0.05)$, while PTX-3 was positively correlated with them $(\mathrm{P}<0.05)$. Combined detection of CTRP9 and PTX-3 was of high value in the diagnosis and prognosis of ACS patients. In addition, CTRP9 and PTX-3 were independent risk factors for the poor prognosis of ACS. Patients with ACS had lower CTRP9 expression and higher PTX-3 expression than those without ACS. Moreover, the combined detection of CTRP9 and PTX-3 can better evaluate the diagnosis and prognosis of ACS patients.
\end{abstract}

Correspondence to: Dr Feng Zhao, Department of Cardiology, Dongying Hospital of Traditional Chinese Medicine, 107 North Second Road, Dongying, Shandong 257000, P.R. China

E-mail: fjq6n3@163.com

*Contributed equally

Key words: C1q/tumor necrosis factor-related protein 9, pentraxin-3, acute coronary syndrome, diagnostic value, prognosis

\section{Introduction}

Acute coronary syndrome (ACS) is a disease with pathological presentation of complete or incomplete occlusion of the coronary artery lumen caused by thrombosis or sudden hemorrhage in atherosclerotic plaques (1). In addition, it is one of the leading causes of acute cardiovascular events clinically at present, posing a serious threat to the life, health and safety of patients (2). However, approximately $15 \%$ of ACS patients cannot be effectively diagnosed at the early stage due to an initially normal electrocardiogram (ECG) (3). Therefore, how to diagnose ACS in a timely and effective manner has important clinical significance for the treatment and prognosis of patients. At present, troponin, myoglobin and brain natriuretic peptide (BNP) are the most commonly used biomarkers in ACS diagnosis. However, these biomarkers are not sensitive to the diagnosis of early ACS $(4,5)$. Thus, finding biomarkers with higher sensitivity is the focus of researches.

Clq/tumor necrosis factor-related protein 9 (CTRP9) is a newly discovered adipocyte factor with similar structure and metabolic regulation function to adiponectin (APN). Moreover, CTRP9 and APN also form heterotrimeric complexes in vitro and in vivo (6). A study revealed that CTRP9 has an inhibitory effect on systemic inflammatory response, and the reduction of its expression is the start of atherosclerosis as well as other diseases (7). Another study revealed that CTRP9 is an independent risk factor for coronary artery disease and predicts the severity of ACS (8). It is considered that the decrease of CTRP9 is proportional to the incidence, mortality and severity of heart failure (9). Plasma pentraxin-3 (PTX-3) is an acute phase reactant protein with similar structure and function to C-reactive protein (CRP), which is highly expressed in coronary plaques (10). PTX-3, secreted by macrophages and neutrophils, promotes the aggregation of monocytes and the formation of atherosclerotic plaques (11).

Therefore, we suspected that the combined detection of CTRP9 and PTX-3 had a high diagnostic value for ACS and could effectively predict the prognosis of patients. However, there are relatively few studies on CTRP9 and PTX-3 in ACS at present, and thus, we conducted a preliminary study. 


\section{Patients and methods}

Patient data. A total of 137 patients (71 males and 66 females) with coronary heart disease (CHD) and chest pain admitted to Yidu Central Hospital of Weifang (Weifang, China) from April 2014 to September 2017 were prospectively recruited. According to the results of coronary angiography, 79 patients with early ACS served as the study group, including 40 with unstable angina pectoris (UAP) (12) and 39 with acute myocardial infarction (AMI) (13). Another 58 patients with non-cardiac chest pain (NCCP) served as the control group. The average age of all the patients was (63.71 \pm 8.27$)$ years. Inclusion criteria were as follows: Patients with early ACS enrolled in the study group and CHD patients without ACS enrolled in the control group were diagnosed by coronary angiography and MRI (14). Exclusion criteria were as follows: Patients with other malignant tumors, systemic immune system diseases, severe liver and kidney dysfunction; pregnancy patients; and NYHA-IV patients. All participants agreed to participate and signed the informed consent form. The present study was approved by the Ethics Committee of Yidu Central Hospital of Weifang (approval no. SDL201404135).

Index detection. Venous blood was collected from all patients within $24 \mathrm{~h}$ after admission and centrifuged at $1,500 \mathrm{x} \mathrm{g}$ for $10 \mathrm{~min}$ to obtain serum at $4^{\circ} \mathrm{C}$. Enzyme-linked immunosorbent assay (ELISA) was used to detect serum CTRP9 [cat. no. RD191180200R; Seebio Biotech (Shanghai) Co., Ltd.] and PTX-3 (product no. kt99347; MSK Biotechnology Co., Ltd.). In addition, an ECG was carried out, and ACS-related serum indexes cardiac troponin I (cTnI) [FDA registration no. (Wuhan) 20182402369; Wuhan Easydiagnosis Biomedicine Co., Ltd.], creatine kinase-MB (CK-MB) [FDA registration no. (Wuhan) 20192402668; Wuhan Easydiagnosis Biomedicine Co., Ltd.] were detected by chemiluminescence, and high-sensitivity C-reactive protein (hs-CRP) by an immunofluorescence analyzer (product no. YM0061114; i-CHROMA) with a kit from Getein Biotech [FDA registration no. (Suzhou) 20152400403]. The patients with ACS were followed up for 3 months, with the end point of the occurrence of cardiovascular events (cardiac death or readmission for ACS).

Statistical analysis. In the present study, the SPSS 18.0 (SPSS, Inc.) was used for statistical analysis. The categorized data were analyzed using the chi-squared test, and the continuous data were expressed as the mean \pm standard deviation. Independent samples t-test was used for comparison between the two groups. GraphPad Prism 6 software (GraphPad Software, Inc.) was used to visualize the data. Pearson's correlation test was applied for correlation analysis, and receiver operating characteristic (ROC) curves were plotted to analyze the diagnostic and prognostic value of CTRP9 and PTX-3 in ACS. The multivariate analysis of prognostic risk factors was carried out with logistic regression model. A value of $\mathrm{P}<0.05$ was considered to indicate a statistically significant difference.

\section{Results}

Comparison of general data. There was no significant difference in sex, age, body mass index (BMI) and basic diseases between the two groups $(\mathrm{P}>0.05)$, as revealed in Table I.
Comparison of the expression of CTRP9 and PTX-3. The expression of CTRP9 in the study group was significantly lower than that in the control group, and the expression of PTX-3 was significantly higher than that in the control group $(\mathrm{P}<0.05)$, as revealed in Table II.

Detection of other relevant indexes. To further analyze the differences between ACS and non-ACS patients, the expression levels of cTnI, CK-MB, and hs-CRP in the two groups were compared. It was revealed that cTnI, CK-MB and hs-CRP in the study group were significantly higher than those in the control group $(\mathrm{P}<0.05)$, as indicated in Fig. 1.

Correlation of serum CTRP9 and PTX-3 with cTnI, CK-MB and hs-CRP. Serum CTRP9 was negatively correlated with cTnI, CK-MB and hs-CRP in ACS $(\mathrm{P}<0.05)$, while PTX-3 was positively correlated with them $(\mathrm{P}<0.05)$, as revealed in Table III and Fig. 2.

Diagnostic values of CTRP9 and PTX-3 in ACS. The sensitivity, specificity, area under curve (AUC), $95 \%$ confidence interval (CI), and cut-off value of CTRP9 for ACS diagnosis were $70.69,72.15 \%, 0.791,0.715-0.867$, and $3.429 \mathrm{mg} / 1$, respectively, and those of PTX-3 were 68.97, 74.68\%, 0.773, $0.692-0.854$, and $0.821 \mu \mathrm{g} / \mathrm{l}$. Whereas the sensitivity, specificity, AUC, and 95\% CI of combined diagnosis of CTRP9 and PTX-3 were 86.21, 77.22\%, 0.876, and 0.813-0.939, respectively. Therefore, although CTRP9 and PTX-3 each had diagnostic value for ACS, the sensitivity and AUC of combined diagnosis were higher than that of single diagnosis, indicating a higher diagnostic value, as revealed in Fig. 3.

Predictive values of CTRP9 and PTX-3 for cardiovascular events in ACS patients. The patients were divided into a cardiovascular event group (36 cases) and a non-cardiovascular event group (43 cases). Comparison of CTRP9 and PTX-3 between the two groups revealed that CTRP9 in the cardiovascular event group was significantly lower than that in the non-cardiovascular event group, and PTX-3 was significantly higher than that in the non-cardiovascular event group (Table IV). Moreover, the predictive values of CTRP9 and PTX-3 for coronary artery events in ACS patients were analyzed, and the combined detection exhibited higher predictive value for poor prognosis of ACS patients (Fig. 4).

Univariate analysis of risk factors for poor prognosis. Univariate analysis revealed that there was no significant difference in sex, age and CK-MB between the cardiovascular event group and non-cardiovascular event group ( $\mathrm{P}>0.05)$, but there were significant differences in hypertension, diabetes, hyperlipidemia, CTRP9, PTX-3, cTnI and hs-CRP $(\mathrm{P}<0.05)$, as revealed in Table V.

Multivariate analysis of risk factors for poor prognosis. According to the results from the univariate analysis, hypertension, diabetes, hyperlipemia, low CTRP9, high PTX-3, high cTnI, and high CK-MB that were significant in univariate analysis were included in multivariate analysis and assigned as variables (Table VI). Multivariate analysis 
Table I. Comparison of general data.

\begin{tabular}{|c|c|c|c|c|}
\hline Factor & Study group $n=79$ & Control group $n=58$ & $\mathrm{t} / \chi^{2}$ & P-value \\
\hline $\operatorname{Sex}[\mathrm{n}(\%)]$ & & & 0.000 & 0.934 \\
\hline Male & $41(51.90)$ & $30(51.72)$ & & \\
\hline Female & $38(48.10)$ & $28(48.28)$ & & \\
\hline Age (years) [n (\%)] & & & 0.007 & 0.931 \\
\hline$\leq 63$ & $36(45.57)$ & $26(44.83)$ & & \\
\hline$>63$ & $43(54.43)$ & $32(55.17)$ & & \\
\hline $\operatorname{BMI}\left(\mathrm{kg} / \mathrm{m}^{2}\right)[\mathrm{n}(\%)]$ & & & 0.032 & 0.858 \\
\hline$\leq 22$ & $41(51.90)$ & $31(53.45)$ & & \\
\hline$>22$ & $38(48.10)$ & $27(46.55)$ & & \\
\hline Hypertension [n (\%)] & & & 0.008 & 0.927 \\
\hline Yes & $51(64.56)$ & $37(63.79)$ & & \\
\hline No & $28(35.44)$ & $21(36.21)$ & & \\
\hline High blood lipid [n (\%)] & & & 0.016 & 0.899 \\
\hline Yes & $40(50.63)$ & $30(51.72)$ & & \\
\hline No & $39(49.37)$ & $28(48.28)$ & & \\
\hline Alanine aminotransferase (IU/l) & $26.34 \pm 1.54$ & $26.29 \pm 1.61$ & 0.184 & 0.854 \\
\hline Aspartate aminotransferase (IU/l) & $21.26 \pm 1.17$ & $21.21 \pm 1.13$ & 0.251 & 0.802 \\
\hline Creatinine $(\mu \mathrm{mol} / \mathrm{l})$ & $64.91 \pm 4.26$ & $64.81 \pm 4.31$ & 0.135 & 0.893 \\
\hline
\end{tabular}

BMI, body mass index.

Table II. Comparison of the expression of CTRP9 and PTX-3.

\begin{tabular}{lccr}
\hline Factor & Study group $\mathrm{n}=79$ & Control group $\mathrm{n}=58$ & $\mathrm{t}$ \\
\hline CTRP9 $(\mathrm{mg} / \mathrm{l})$ & $3.31 \pm 0.19$ & $3.53 \pm 0.20$ & 6.549 \\
PTX-3 $(\mu \mathrm{g} / \mathrm{l})$ & $0.89 \pm 0.15$ & $0.75 \pm 0.12$ & 5.861 \\
\hline
\end{tabular}

CTRP9, C1q/tumor necrosis factor-related protein 9; PTX-3, pentraxin-3.

Table III. Correlation of serum CTRP9 and PTX-3 with cTnI, CK-MB and hs-CRP.

\begin{tabular}{lccccr}
\hline & \multicolumn{2}{c}{ CTRP9 } & & \multicolumn{2}{c}{ PTX-3 } \\
\cline { 2 - 3 } \cline { 5 - 6 } Index & $\mathrm{r}$ & P-value & & $\mathrm{r}$ & P-value \\
\hline cTnI & -0.735 & $<0.001$ & & 0.626 & $<0.001$ \\
CK-MB & -0.723 & $<0.001$ & & 0.672 & $<0.001$ \\
hs-CRP & -0.686 & $<0.001$ & & 0.774 & $<0.001$ \\
\hline
\end{tabular}

CTRP9, C1q/tumor necrosis factor-related protein 9; PTX-3, pentraxin-3; cTnI, cardiac troponin I; CK-MB, creatine kinase-MB; hs-CRP, high-sensitivity C-reactive protein.

was carried out using logistic regression model to analyze the risk factors of cardiovascular events. The results revealed that hypertension, hyperlipemia, low CTRP9, high PTX-3 were independent risk factors for adverse cardiovascular events (Table VII).

\section{Discussion}

ACS is a disease with complicated physiological and pathological processes whose diagnosis and prognosis cannot be predicted by a single index (15). Several myocardial indicators are released into the blood only after necrosis of myocardial cells. If there is still no obvious abnormality in the ECG, it is easy to delay the diagnosis, and therefore delay timely treatment of patients (16). Therefore, more laboratory parameters are required for the diagnosis of ACS.

In the present study, CTRP9 and PTX-3 were selected to diagnose CHD patients with ACS. It was determined that serum CTRP9 in patients with ACS was significantly lower than that in patients without ACS, while the expression of PTX-3 was significantly higher than that of patients without ACS. A previous study demonstrated that CTRP9 was highly expressed in the serum of cardiovascular disease patients with high plaque stability (17). Another study suggested that it contributed to the enhancement of plaque stability by reducing the expression of pro-inflammatory factors in macrophages (7), which confirms our conclusion concerning the low expression of CTRP9 in ACS. However, PTX-3, an inflammatory protein homologous to CRP, 

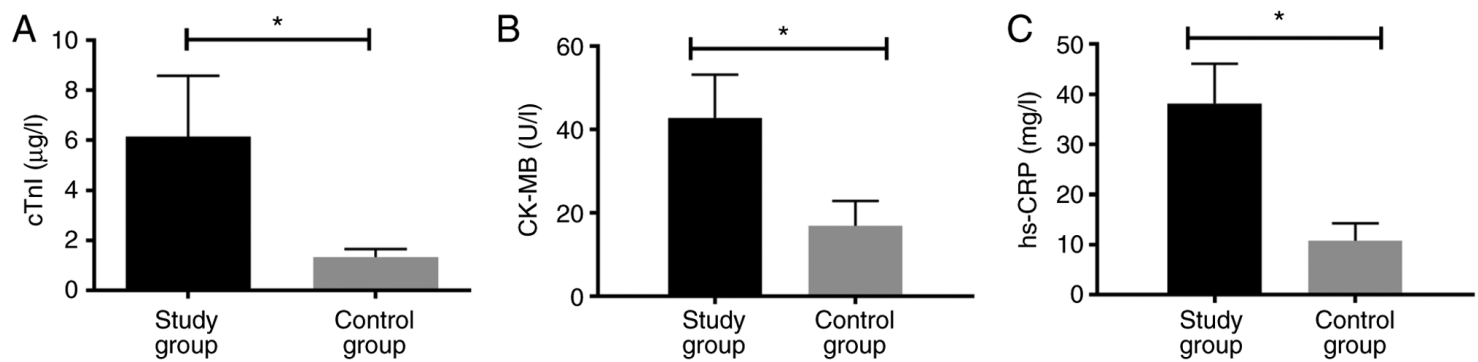

Figure 1. Detection of cTnI, CK-MB and hs-CRP. (A) cTnI expression in the study group was significantly higher than that in the control group. (B) CK-MB expression in the study group was significantly higher than that in the control group. (C) hs-CRP expression in the study group was significantly higher than that in the control group. ${ }^{\text {P }}<0.05$. cTnI, cardiac troponin I; CK-MB, creatine kinase-MB; hs-CRP, high-sensitivity C-reactive protein.
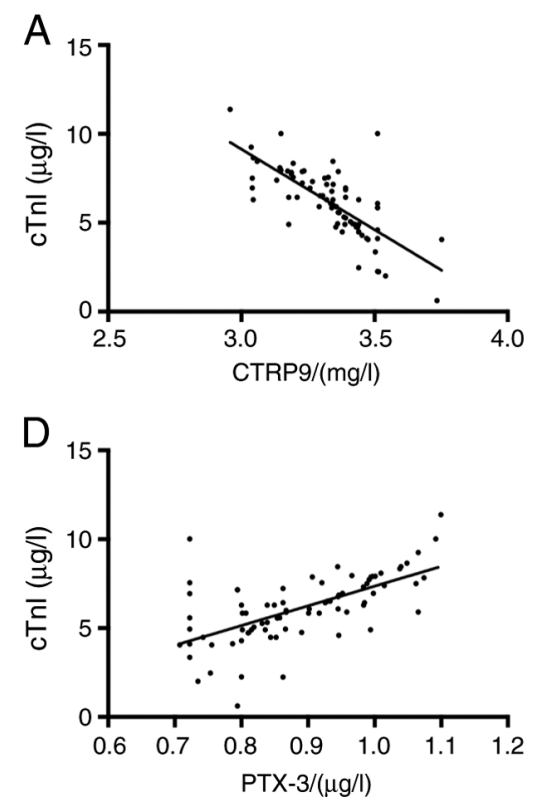
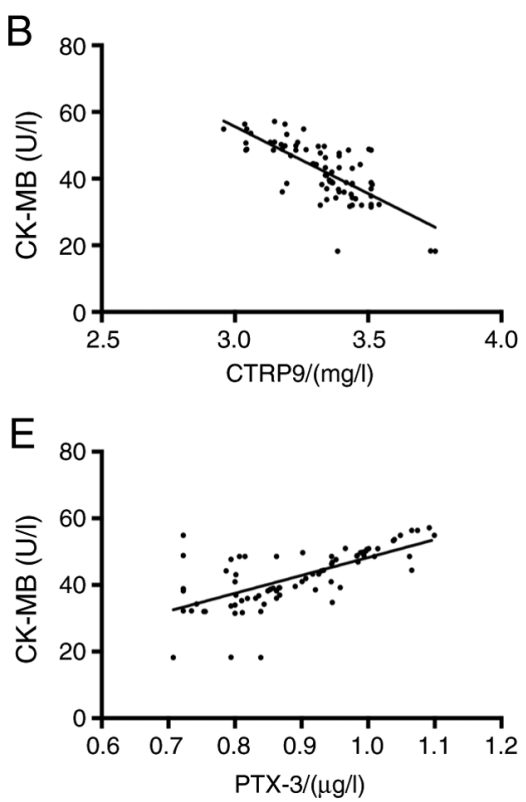
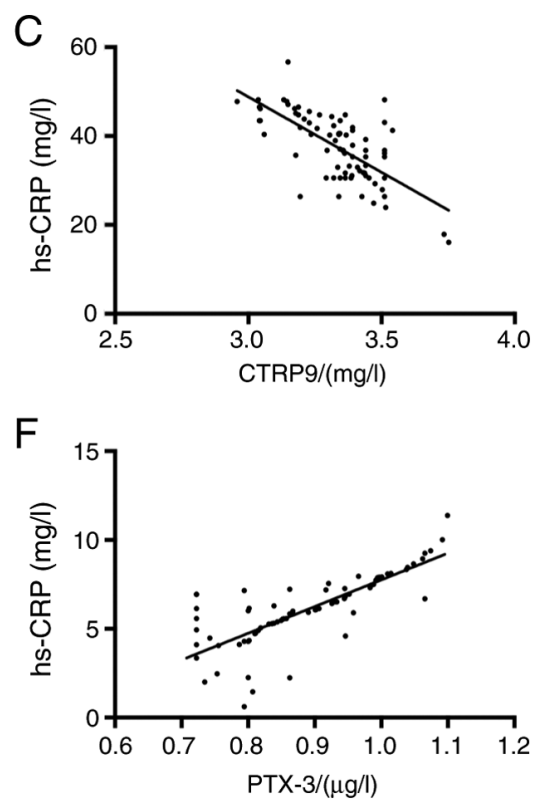

Figure 2. Correlation of serum CTRP9 and PTX-3 with cTnI, CK-MB and hs-CRP. (A) CTRP9 and cTnI are negatively correlated ( $\mathrm{r}=-0.735$; P $<0.05$ ). (B) CTRP9 and CK-MB are negatively correlated ( $\mathrm{r}=-0.723$; $\mathrm{P}<0.05)$. (C) CTRP9 and hs-CRP are negatively correlated $(\mathrm{r}=-0.686$; $\mathrm{P}<0.05)$. (D) PTX-3 and cTnI are positively correlated $(\mathrm{r}=0.626 ; \mathrm{P}<0.05)$. (E) PTX-3 and CK-MB are positively correlated $(\mathrm{r}=0.672 ; \mathrm{P}<0.05)$. (F) PTX-3 and hs-CRP are positively correlated ( $\mathrm{r}=0.774 ; \mathrm{P}<0.05)$. CTRP9, Clq/tumor necrosis factor-related protein 9; PTX-3, pentraxin-3; cTnI, cardiac troponin I; CK-MB, creatine kinase-MB; hs-CRP, hypersensitive C-reactive protein.
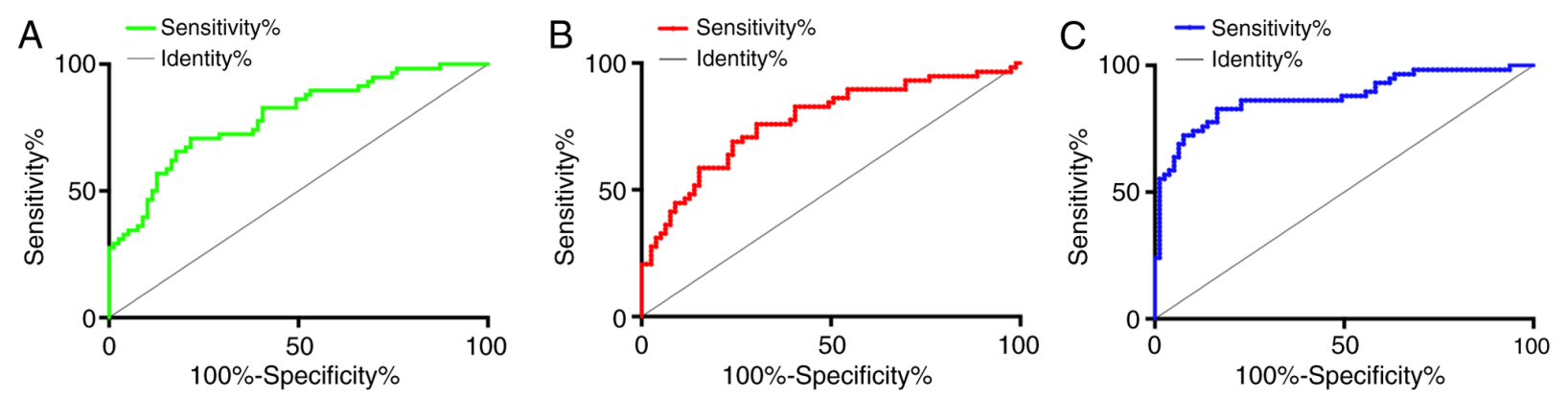

Figure 3. Diagnostic values of CTRP9 and PTX-3 in ACS. (A) The sensitivity, specificity, AUC, 95\% CI, and cut-off value of CTRP9 for ACS diagnosis were 70.69, 72.15\%, 0.791, 0.715-0.867 and $3.429 \mathrm{mg} / 1$ respectively. (B) The sensitivity, specificity, AUC, $95 \%$ CI, and cut-off value of PTX-3 for ACS diagnosis were $68.97,74.68 \%, 0.773,0.692-0.854$ and $0.821 \mu \mathrm{g} / 1$, respectively. (C) The sensitivity, specificity, AUC, and 95\% CI of combined of CTRP9 and PTX-3 for ACS diagnosis were 86.21, 77.22\%, 0.876 and 0.813-0.939 respectively. CTRP9, C1q/tumor necrosis factor-related protein 9; PTX-3, pentraxin-3; ACS, acute coronary syndrome; AUC, area under curve; CI, confidence interval.

significantly increases after infection and its peak expression is higher than of CRP, which indicates that PTX-3 may better reflect the inflammation of blood vessels than CRP $(18,19)$. Other cardiovascular disease-related factors (cTnI, CK-MB and hs-CRP) were detected, and the results revealed that the factors in ACS patients were significantly higher than those in the controls. cTnI, 
Table IV. Comparison of serum CTRP9 and PTX-3 in patients with different prognosis.

\begin{tabular}{|c|c|c|c|c|}
\hline & Cardiovascular event group $(n=36)$ & Non-cardiovascular event group $(n=43)$ & $\mathrm{t}$ & P-value \\
\hline CTRP9/(mg/l) & $3.23 \pm 0.11$ & $3.36 \pm 0.10$ & 5.498 & $<0.001$ \\
\hline PTX-3/( $\mu \mathrm{g} / 1)$ & $0.99 \pm 0.10$ & $0.84 \pm 0.09$ & 7.013 & $<0.001$ \\
\hline
\end{tabular}

Table V. Univariate analysis of the poor prognosis in patients with ACS.

\begin{tabular}{|c|c|c|c|c|}
\hline Factor & $\begin{array}{c}\text { Cardiovascular } \\
\text { event group }(n=36)\end{array}$ & $\begin{array}{l}\text { Non-cardiovascular } \\
\text { event group }(n=43)\end{array}$ & $t / \chi^{2}$ & P-value \\
\hline $\operatorname{Sex}[\mathrm{n},(\%)]$ & & & 0.096 & 0.757 \\
\hline Male & $18(50.00)$ & $23(53.48)$ & & \\
\hline Female & $18(50.00)$ & $20(46.52)$ & & \\
\hline Age (years) & $63.68 \pm 8.16$ & $63.75 \pm 8.22$ & 0.049 & 0.961 \\
\hline Hypertension [n, (\%)] & $28(77.78)$ & $23(53.49)$ & 5.052 & 0.025 \\
\hline Diabetes $[\mathrm{n},(\%)]$ & $27(75.00)$ & $20(46.51)$ & 6.599 & 0.010 \\
\hline High blood lipid [n, (\%)] & $25(69.44)$ & $15(34.88)$ & 9.364 & 0.002 \\
\hline CTRP9 (mg/l) & $3.23 \pm 0.11$ & $3.36 \pm 0.10$ & 5.498 & $<0.001$ \\
\hline PTX-3 $(\mu \mathrm{g} / \mathrm{l})$ & $0.99 \pm 0.10$ & $0.84 \pm 0.09$ & 7.013 & $<0.001$ \\
\hline $\mathrm{cTnI}(\mu \mathrm{g} / 1)$ & $7.33 \pm 1.26$ & $5.52 \pm 1.13$ & 6.728 & $<0.001$ \\
\hline CK-MB (U/1) & $49.96 \pm 7.34$ & $48.91 \pm 6.63$ & 0.668 & 0.506 \\
\hline hs-CRP (mg/l) & $43.51 \pm 4.68$ & $36.92 \pm 3.47$ & 7.176 & $<0.001$ \\
\hline
\end{tabular}

ACS, acute coronary syndrome; CTRP9, C1q/tumor necrosis factor-related protein 9; PTX-3, pentraxin-3; cTnI, cardiac troponin I; CK-MB, creatine kinase-MB; hs-CRP, high-sensitivity C-reactive protein.

Table VI. Assignment table.

\begin{tabular}{lc}
\hline Factor & Assignment \\
\hline Hypertension & Yes $=1, \mathrm{No}=2$ \\
Diabetes & Yes $=1, \mathrm{No}=2$ \\
High blood lipid & Yes $=1, \mathrm{No}=2$ \\
CTRP9 & $>3.31 \mathrm{mg} / \mathrm{l}=1, \leq 3.31 \mathrm{mg} / \mathrm{l}=2$ \\
PTX-3 & $>0.89 \mu \mathrm{g} / \mathrm{l}=1, \leq 0.89 \mu \mathrm{g} / \mathrm{l}=2$ \\
cTnI & $>6.15 \mu \mathrm{g} / \mathrm{l}=1, \leq 6.15 \mu \mathrm{g} / \mathrm{l}=2$ \\
hs-CRP & $>38.19 \mathrm{mg} / \mathrm{l}=1, \leq 38.19 \mathrm{mg} / \mathrm{l}=2$
\end{tabular}

CTRP9, Clq/tumor necrosis factor-related protein 9; PTX-3, pentraxin-3; cTnI, cardiac troponin I; hs-CRP, hypersensitive C-reactive protein.

CK-MB and hs-CRP are sensitive factors to myocardial injury. cTnI has been reported to have a high sensitivity to myocardial infarction, however, due to its insignificant changes in the early stage of ACS, it can only be used to evaluate the severity of myocardial infarction (20). Similarly, although highly sensitive to myocardial infarction, CK-MB has poor specificity for myocardial injury because it is also increased in patients with diabetes and skeletal muscle trauma (21). As the most sensitive factor to the inflammatory response, hs-CRP can predict the occurrence of ACS, but still with poor diagnostic specificity (22). Therefore, the correlation of serum CTRP9 and PTX-3 with cTnI, CK-MB and hs-CRP in ACS patients was analyzed. It was revealed that serum CTRP9 was negatively correlated with cTnI, CK-MB and hs-CRP in ACS patients, while PTX-3 was positively correlated with them, which further suggested that there was a close relationship between CTRP9, PTX-3 and the incidence of ACS.

Subsequently, in order to further clarify the role of CTRP9 and PTX-3 in the pathogenesis and prognosis of ACS, we separately and jointly detected CTRP9 and PTX-3. According to the ROC curve, a common tool for evaluating the diagnostic efficiency, the AUC of combined detection of CTRP9 and PTX-3 for ACS diagnosis and poor prognosis prediction was 0.876 and 0.894 respectively, which indicated that the combined detection had a high predictive value for the onset and prognosis of ACS. Furthermore, a multivariate analysis of risk factors for cardiovascular adverse events was carried out in order to analyze the influencing factors that affect the prognosis of ACS patients. The results revealed that hypertension, hyperlipidemia, low CTRP9 and high PTX-3 were independent risk factors for cardiovascular adverse events in ACS patients. At present, the application of CTRP9 and PTX-3 in ACS diagnosis has seldom been studied. Only a few studies have revealed that low-CTRP9 or high-PTX-3 expression is an important indicator for the pathogenesis of ACS $(23,24)$. However, CTRP9 has been revealed to interfere with insulin-mediated glucose uptake by activating adenosine, thereby inhibiting the proliferation of smooth muscle cells and vascular injury (25). The expression of CTRP9 significantly decreased when patients suffered from myocardial cell injury, 
Table VII. Multivariate analysis of the poor prognosis in patients with ACS.

\begin{tabular}{lcccccc}
\hline Factor & $\beta$ & SE & Wald & OR & $95 \%$ CI & P-value \\
\hline Hypertension & 0.067 & 0.010 & 3.173 & 1.231 & $1.082-1.403$ & $<0.01$ \\
Diabetes & 0.009 & 0.053 & 3.783 & 1.431 & $1.042-1.767$ & 0.052 \\
High blood lipid & 0.028 & 0.114 & 0.057 & 1.420 & $1.109-2.421$ & $<0.01$ \\
CTRP9 & 0.199 & 0.425 & 7.183 & 1.221 & $1.175-4.418$ & $<0.01$ \\
PTX-3 & 0.205 & 0.315 & 4.103 & 3.017 & $1.031-8.792$ & $<0.01$ \\
cTnI & 0.004 & 0.003 & 0.289 & 1.659 & $1.029-4.121$ & 0.582 \\
hs-CRP & 0.124 & 0.321 & 0.132 & 1.312 & $1.052-2.614$ & 0.730 \\
\hline
\end{tabular}

ACS, acute coronary syndrome; CTRP9, C1q/tumor necrosis factor-related protein 9; PTX-3, pentraxin-3; cTnI, cardiac troponin I; hs-CRP, hypersensitive C-reactive protein; $\beta$, beta regression coefficient; SE, standard error; OD, odds ratio; CI, confidence interval.
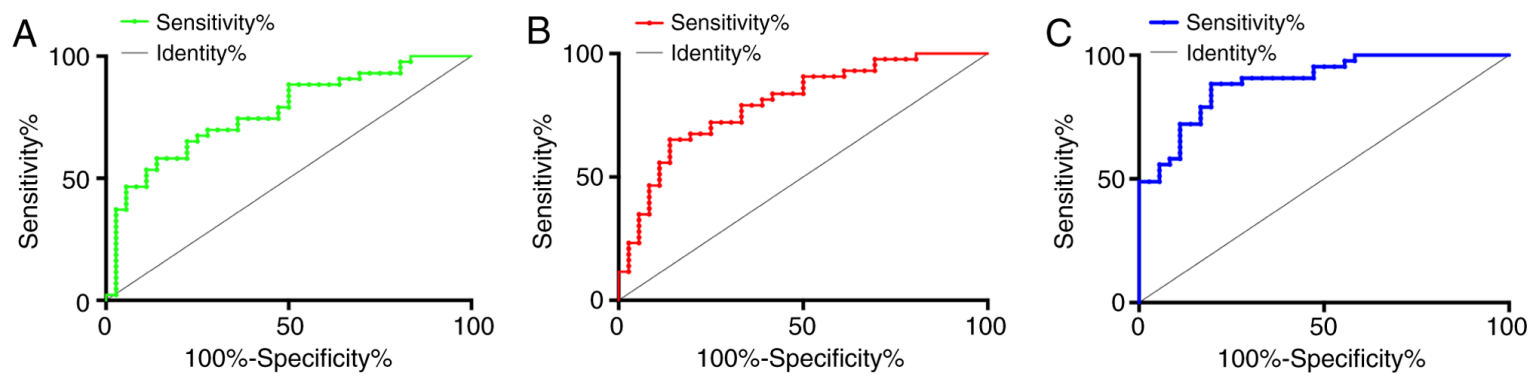

Figure 4. Predictive values of CTRP9 and PTX-3 for cardiovascular events in ACS. (A) The sensitivity, specificity, and AUC of CTRP9 in cardiovascular event prediction were $72.09,63.89 \%$, and 0.770 , respectively. (B) The sensitivity, specificity, and AUC of PTX-3 in cardiovascular event prediction were $74.42,66.67 \%$, and 0.799, respectively. (C) The sensitivity, specificity, and AUC of combined of CTRP 9 and PTX-3 in cardiovascular event prediction were 88.37, 77.78\%, and 0.894, respectively. CTRP9, C1q/tumor necrosis factor-related protein 9; PTX-3, pentraxin-3; ACS, acute coronary syndrome; AUC, area under curve.

thus CTRP9 can better reflect myocardial injury. Myocardial infarction area and apoptosis of myocardial cells in mice were revealed to be increased after CTRP9 knockout, thus it is inferred that low expression of CTRP9 may lead to poor prognosis in ACS mice (26). Moreover, there is evidence that CTRP9 mediates protective effects in cardiomyocytes via AMPK- and adiponectin receptor-mediated induction of anti-oxidant response (27). PTX-3 has been reported to promote the formation of atherosclerotic plaque by accelerating the aggregation of monocytes, and the increase of serum PTX-3 indicates poor prognosis of patients with ACS (28). In addition, it always leads to the increase of fibrinogen and induces a hypercoagulable state of blood vessels; moreover, its interaction with inflammatory reaction may cause unstable atherosclerotic plaque, thereby resulting in the occurrence of ACS; therefore, PTX-3 is considered to be an important predictor of ACS (29). There is a study that specifically revealed that the inclusion of PTX-3 improved the predictive model of cardiovascular complications in patients with myocardial infarction (30). All the aforementioned studies confirmed the conclusions of the present study.

To sum up, CTRP9 was revealed to be lowly expressed and PTX-3 to be highly expressed in the serum of patients with ACS. Combined detection of CTRP9 and PTX-3 can better evaluate and predict the onset and prognosis of ACS. However, there are still several limitations in the present study. Firstly, due to the lack of long-term follow-up, it is impossible to determine the correlation of CTRP9 and PTX-3 with the death of patients. Secondly, the high cardiovascular events in the present study may be a random outcome due to the small sample size included. In a follow-up study, the sample size will be expanded for further exploration. Thirdly, the absence of healthy participants as controls may lead to a lack of data for the assessment of CTRP9 and PTX-3 in CHD. Although this is not our main objective, it will be further explored in future studies.

\section{Acknowledgements}

Not applicable.

\section{Funding}

The present study was supported by the project: Association between serum CTRP9 and the occurrence, severity and short-term and long-term prognosis of coronary heart disease (grant no. 2018X05187) from Dongying Hospital of Traditional Chinese Medicine (Dongying, China).

\section{Availability of data and materials}

The datasets used and/or analyzed during the current study are available from the corresponding author on reasonable request.

\section{Authors' contributions}

NaJ and SZ designed the study and drafted the manuscript. GW and NiJ were responsible for the collection and analysis 
of the experimental data. $\mathrm{HW}$ and $\mathrm{FZ}$ revised the manuscrip critically for important intellectual content. All authors read and approved the final manuscript.

\section{Ethics approval and consent to participate}

The present study was approved by the Ethics Committee of Yidu Central Hospital of Weifang, China. Patients who participated in this research, signed the informed consent and had complete clinical data. Signed written informed consents were obtained from the patients and/or guardians.

\section{Patient consent for publication}

Not applicable.

\section{Competing interests}

The authors declare that they have no competing interests.

\section{References}

1. Cuisset T, Deharo P, Quilici J, Johnson TW, Deffarges S, Bassez C, Bonnet G, Fourcade L, Mouret JP, Lambert M, et al: Benefit of switching dual antiplatelet therapy after acute coronary syndrome: The TOPIC (timing of platelet inhibition after acute coronary syndrome) randomized study. Eur Heart J 38: 3070-3078, 2017.

2. Kai ME and Lindahl B: Application of cardiac troponin in cardiovascular diseases other than acute coronary syndrome. Clin Chem 63: 223-235, 2017.

3. Hamm CW, Bertrand M and Braunwald E: Acute coronary syndrome without ST elevation: Implementation of the new guidelines. Lancet 358: 1533-1538, 2001.

4. Katus H, Ziegler A, Ekinci O, Giannitsis E, Stough WG, Achenbach S, Blankenberg S, Brueckmann M, Collinson P, Comaniciu D, et al: Early diagnosis of acute coronary syndrome. Eur Heart J 38: 3049-3055, 2017.

5. Mishra B, Pandey S, Niraula SR, Rai BK, Karki P, Baral N and Lamsal M: Utility of ischemia modified albumin as an early marker for diagnosis of acute coronary syndrome. J Nepal Health Res Counc 16: 16-21, 2018

6. Peterson JM, Wei Z, Seldin MM, Byerly MS, Aja S and Wong GW: CTRP9 transgenic mice are protected from diet-induced obesity and metabolic dysfunction. Am J Physiol Regul Integr Comp Physiol 305: R522-R533, 2013.

7. Li J, Zhang P, Li T, Liu Y, Zhu Q, Chen T, Liu T, Huang C, Zhang J, Zhang Y and Guo Y: CTRP9 enhances carotid plaque stability by reducing pro-inflammatory cytokines in macrophages. Biochem Biophys Res Commun 458: 890-895, 2015.

8. Liu T, Guo Y, Li T, Zhang J, Li J and Zhang P: Association of serum CTRP9 and APN levels with acute coronary syndrome. J Shandong Univ 52: 58-62, 2014.

9. Gao C, Zhao S, Lian K, Mi B, Si R, Tan Z, Fu F, Wang S, Wang R, Ma X and Tao L: C1q/TNF-related protein 3 (CTRP3) and 9 (CTRP9) concentrations are decreased in patients with heart failure and are associated with increased morbidity and mortality. BMC Cardiovasc Disord 19: 139, 2019.

10. George M, Shanmugam E, Srivatsan V, Vasanth K, Ramraj B, Rajaram M, Jena A, Sridhar A, Chaudhury M and Kaliappan I: Value of pentraxin-3 and galectin-3 in acute coronary syndrome: A short-term prospective cohort study. Ther Adv Cardiovasc Dis 9: 275, 2015.

11. Barazzoni R, Aleksova A, Carriere C, Cattin MR, Zanetti M, Vinci P, Stolfo D, Guarnieri G and Sinagra G: Obesity and high waist circumference are associated with low circulating pentraxin-3 in acute coronary syndrome. Cardiovasc Diabetol 12: 167-167, 2013.

12. Anderson JL, Adams CD, Antman EM, Bridges CR, Califf RM, Casey DE Jr, Chavey WE II, Fesmire FM, Hochman JS, Levin TN, et al: 2012 ACCF/AHA focused update incorporated into the ACCF/AHA 2007 guidelines for the management of patients with unstable angina/non-ST-elevation myocardial infarction: A report of the American College of Cardiology Foundation/American Heart Association Task Force on Practice Guidelines. J Am Coll Cardiol 61: e179-e347, 2013.
13. Mehrshad V: State-of-the-art diagnosis of myocardial infarction. Diagnosis (Berl) 3: 137-142, 2016.

14. Panovský R, Borová J, Pleva M, Feitová V, Novotný P, Kincl V, Holeček T, Meluzín J, Sochor O and Štěpánová R: The unique value of cardiovascular magnetic resonance in patients with suspected acute coronary syndrome and culprit-free coronary angiograms. BMC Cardiovasc Disord 17: 170, 2017.

15. Chacko S, Haseeb S, Glover BM, Wallbridge D and Harper A: The role of biomarkers in the diagnosis and risk stratification of acute coronary syndrome. Future Sci Oa 4: FSO251, 2018.

16. Wang W, Ren D, Wang CS and Yao HC: Nardilysin: A potential biomarker for the early diagnosis of acute coronary syndrome. Int J Cardiol 265: 39, 2018.

17. Choi KM, Hwang SY, Hong HC, Choi HY, Yoo HJ, Youn BS, Baik SH and Seo HS: Implications of C1q/TNF-related protein-3 (CTRP-3) and progranulin in patients with acute coronary syndrome and stable angina pectoris. Cardiovasc Diabetol 13: $14,2014$.

18. Gustin C, Delaive E, Dieu M, Calay D and Raes M: Up-regulation of pentraxin-3 in human endothelial cells after lysophosphatidic acid exposure. Arterioscler Thromb Vasc Biol 28: 491-497, 2008.

19. Kotooka N, Inoue T, Aoki S, Anan M, Komoda H and Node K: Prognostic value of pentraxin 3 in patients with chronic heart failure. Int J Cardiol 130: 19-22, 2008

20. Hasic S, Kadic D, Kiseljakovic E, Jadric R and Spahic E: Serum uric acid could differentiate acute myocardial infarction and unstable angina pectoris in hyperuricemic acute coronary syndrome patients. Med Arch 71: 115-118, 2017.

21. Natsukawa T, Maeda N, Fukuda S, Yamaoka M, Fujishima Y, Nagao H, Sato F, Nishizawa H, Sawano H, Hayashi Y, et al: Significant association of serum adiponectin and creatine kinase-MB levels in ST-segment elevation myocardial infarction. J Atheroscler Thromb 24: 793-803, 2017.

22. Hong YJ, Jeong MH, Choi YH, Cho SH, Hwang SH, Ko JS, Lee MG, Park KH, Sim DS, Yoon NS, et al: Relation between high-sensitivity $\mathrm{C}$-reactive protein and coronary plaque components in patients with acute coronary syndrome: Virtual histology-intravascular ultrasound analysis. Korean Circ J 41: 440-446, 2011

23. Cheng GD: RDW value for assessment of the severity of acute coronary syndrome and its correlation with serum indexes. J Hainan Med Univ, 2016.

24. Kambara T, Ohashi K, Shibata R, Ogura Y,Maruyama S,Enomoto T, Uemura Y, Shimizu Y, Yuasa D, Matsuo K, et al: CTRP9protein protects against myocardial injury following ischemia-reperfusion through AMP-activated protein kinase (AMPK)-dependent mechanism. J Biol Chem 287: 18965-18973, 2012.

25. Uemura Y, Shibata R, Ohashi K, Enomoto T, Kambara T, Yamamoto T, Ogura Y, Yuasa D, Joki Y, Matsuo K, et al: Adipose-derived factor CTRP9atenuates vascular smooth muscle cell proliferation and neointimal formation. FASEB J 27: 25-33, 2013

26. Kambara T, Shibata R, Ohashi K, Matsuo K, Hiramatsu-Ito M, Enomoto T, Yuasa D, Ito M, Hayakawa S, Ogawa H, et al: C1q/tumor necrosis factor-related protein 9 protects against acute myocardial injury through an adiponectin receptor I-AMPK-dependent mechanism. Mol Cel Biol 35: 2173-2185, 2015.

27. Niemann B, Li L, Siegler D, Siegler BH, Knapp F, Hanna J, Aslam M, Kracht M, Schulz R and Rohrbach S: CTRP9 mediates protective effects in cardiomyocytes via AMPK- and adiponectin receptor-mediated induction of anti-oxidant response. Cells 9: 1229,2020

28. Eggers KM, Armstrong PW, Califf RM, Johnston N, Simoons ML, Venge $\mathrm{P}$ and James S: Clinical and prognostic implications of circulating pentraxin 3 levels in non ST-elevation acute coronary syndrome. Clin Biochem 46: 1655-1659, 2013.

29. Inoue K, Sugiyama A, Reid PC, Ito Y, Miyauchi K, Mukai S, Safara M, Miyamoto K, Satoh H, Kohno I, et al: Establishmen of a high sensitivity plasma assay for human pentraxin 3 as a marker for unstable angina pectoris. Arterioscler Thromb Vasc Biol 27: 161-167, 2007.

30. Khamitova AF, Lakman IA, Akhmetvaleev RR, Tulbaev EL, Gareeva DF, Zagidullin SZ and Zagidullin NS: Multifactor predictive model in patients with myocardial infarction based on modern biomarkers. Kardiologiia 60: 14-20, 2020 (In Russian).

This work is licensed under a Creative Commons

Attribution-NonCommercial-NoDerivatives 4.0 International (CC BY-NC-ND 4.0) License. 